Supporting information for

\title{
A Nano-Segregated Polymeric Film Exhibiting High Ionic Conductivities
}

Kenji Kishimoto, Tomoyuki Suzawa, Tomoki Yokota, Tomohiro Mukai, Hiroyuki Ohno, and Takashi Kato*

General. NMR spectra were recorded using a JEOL JNM-LA400 at $400 \mathrm{MHz}$ for ${ }^{1} \mathrm{H}$ NMR and at $100 \mathrm{MHz}$ for ${ }^{13} \mathrm{C} \mathrm{NMR}$ in $\mathrm{CDCl}_{3}$. Chemical shifts of ${ }^{1} \mathrm{H}$ and ${ }^{13} \mathrm{C} \mathrm{NMR}$ signals were quoted to internal standard $\mathrm{Me}_{4} \mathrm{Si}(\delta=0.00)$ and $\mathrm{CDCl}_{3}(\delta=77.00)$ respectively, and expressed by chemical shifts in ppm $(\delta)$, multiplicity, coupling constant $(\mathrm{Hz})$, and relative intensity. Elemental analyses were carried out on a Perkin-Elmer CHNS/O 2400 apparatus. Mass spectra were recorded on a JEOL JMS-AX505H spectrometer. Liquid-crystalline phases were determined with an Olympus BX51 optical polarizing microscope equipped with a Mettler FP82 HT hot-stage. Differential scanning calorimetry (DSC) measurements were conducted with a NETZSCH DSC 204 Phoenix ${ }^{\circledR}$ differential scanning calorimeter operated at a scanning rate of $10{ }^{\circ} \mathrm{C} \min ^{-1}$ on heating and cooling. Gel permeation chromatography was performed with a TOSOH HLC-8220 GPC instrument equipped with two TSKgel G3000H $\mathrm{HL}_{\mathrm{X}}$ columns connected in series, a TOSOH SD-8020 degasser, a RI detector (TOSOH RI-8020), and a TOSOH CO-8020 column oven $\left(40{ }^{\circ} \mathrm{C}\right)$. X-ray scattering measurements were carried out on a Rigaku RINT-2500 system using $\mathrm{CuK} \alpha$ radiation.

Materials. All reagents of the highest quality were purchased from Aldrich, Kanto, Tokyo Kasei, or Wako, and were used without further purification. Unless otherwise noted, all of the reactions were carried out under an argon atmosphere in dry solvents purchased from Kanto.

2,3-Difluoro-(tert-butyldimethylsilyloxy)benzene. Imidazole $(5.24 \mathrm{~g}, 76.9 \mathrm{mmol})$ was added to a solution of 2,3-difluorophenol $(5.00 \mathrm{~g}, \quad 38.4 \mathrm{mmol})$ and tert-butyldimethylchlorosilane (TBSCl) $(8.70 \mathrm{~g}, 57.7 \mathrm{mmol})$ in $N, N$-dimethylformamide (DMF) $(20 \mathrm{~mL})$. The mixture was stirred at room temperature for 3 hours. The resulting mixture was diluted with diethyl ether and water. The organic phase was separated and the aqueous phase was extracted with diethyl ether three times. The etherial extracts were successively washed with a saturated aqueous $\mathrm{NH}_{4} \mathrm{Cl}$ solution, water, a saturated aqueous $\mathrm{NaCl}$ solution, dried over $\mathrm{MgSO}_{4}$, and filtered. The solvent was removed in vacuo. Purification by column chromatography (eluent: hexane) afforded 
2,3-difluoro-(tert-butyldimethylsilyloxy)benzene $(9.12 \mathrm{~g}, 37.3 \mathrm{mmol})$ in a yield of $97 \%$ as a colorless oil. ${ }^{1} \mathrm{H}$ NMR (400 MHz): $\delta=0.20(\mathrm{~s}, 6 \mathrm{H}), 1.00(\mathrm{~s}, 9 \mathrm{H}), 6.66-6.78(\mathrm{~m}, 2 \mathrm{H})$, 6.86-6.92 (m. 1H). ${ }^{13} \mathrm{C} \mathrm{NMR} \mathrm{(100} \mathrm{MHz):} \delta=-4.8(\mathrm{~s}), 18.2(\mathrm{~s}), 25.4(\mathrm{~s}), 109.6$ (d, $\left.J=17 \mathrm{~Hz}\right)$, $117.2(\mathrm{~d}, J=3 \mathrm{~Hz}), 122.8$ (dd, $J=5,9 \mathrm{~Hz}), 143.4$ (dd, $J=13,245 \mathrm{~Hz}), 144.6$ (d, $J=10 \mathrm{~Hz})$, $151.6(\mathrm{dd}, J=11,247 \mathrm{~Hz}) . \mathrm{MS} \mathrm{m} / \mathrm{z}$ (rel intensity) $244\left(\mathrm{M}^{+}\right.$; 25), 233 (3), 218 (10), 211 (7), 208 (23), 188 (10), 187 (42), 149 (25), 117 (13), 93 (13), 88 (21), 77 (57), 70 (21), 61 (100).

4-tert-Butyldimethylsilyloxy-2,3-difluorophenylboronic acid. n-Butyllithium (1.54 mol

$\left.\mathrm{L}^{-1}, 25 \mathrm{~mL}, 38.5 \mathrm{mmol}\right)$ was added dropwise to a stirred cooled solution $\left(-78{ }^{\circ} \mathrm{C}\right)$ of 2,3-difluoro-(tert-butyldimethylsilyloxy)benzene $(8.96 \mathrm{~g}, 36.6 \mathrm{mmol})$ in tetrahydrofuran (THF) $(100 \mathrm{~mL})$. The reaction mixture was maintained under these conditions for 2.5 hours and then a previously cooled solution of trimethyl borate $(8.55 \mathrm{~g}, 82.3 \mathrm{mmol})$ in THF $(40 \mathrm{~mL})$ was added dropwise at $-78{ }^{\circ} \mathrm{C}$. The reaction mixture was allowed to warm to room temperature overnight and then stirred for 1 hour with 5\% hydrochloric acid $(120 \mathrm{~mL})$. The product was extracted into diethyl ether, and the combined ethereal extracts were washed with water and a saturated aqueous $\mathrm{NaCl}$ solution, dried over $\mathrm{MgSO}_{4}$, and filtered. The solvent was removed in vacuo. The residue was purified by column chromatography (eluent: chloroform $\left(\mathrm{CHCl}_{3}\right) /$ methanol $\left.=20 / 1\right)$ to give 4-tert-butyldimethylsilyloxy-2,3-difluorophenylboronic acid (8.18 g, $28.3 \mathrm{mmol})$ in $78 \%$ yield as a white solid. ${ }^{1} \mathrm{H}$ NMR $(400 \mathrm{MHz}): \delta=0.25(\mathrm{~s}, 6 \mathrm{H})$, $1.02(\mathrm{~s}, 9 \mathrm{H}), 4.84(\mathrm{~s}, 1 \mathrm{H}), 4.86(\mathrm{~s}, 1 \mathrm{H}), 6.73-6.80(\mathrm{~m}, 1 \mathrm{H}), 7.69-7.73(\mathrm{~m}, 1 \mathrm{H}) .{ }^{13} \mathrm{C} \mathrm{NMR}(100$ MHz): $\delta=18.4$ (s), 25.5 (s), $131.4(\mathrm{dd}, J=4.9,17 \mathrm{~Hz}), 143.2(\mathrm{dd}, J=15,246 \mathrm{~Hz}), 149.5$ (dd, $J=3.4,9.6 \mathrm{~Hz}), 157.4(\mathrm{dd}, J=10,257 \mathrm{~Hz}) . \mathrm{MS} \mathrm{m} / \mathrm{z}$ (rel intensity) $244\left(\mathrm{M}^{+}-\mathrm{B}(\mathrm{OH})_{2} ; 3\right), 187$ (41), 109 (13), 91 (26), 81 (18), 78 (8), 77 (100).

4-Bromo-4'-(6-hydroxylhexyloxy)biphenyl. A mixture of 4-bromo-4'-hydroxybiphenyl (3.00 g, $12.0 \mathrm{mmol})$, 6-chloro-1-hexanol (1.48 g, $10.8 \mathrm{mmol})$, tetrabutylammonium iodide (TBAI) $\left(8.94 \times 10^{-1} \mathrm{~g}, 2.42 \mathrm{mmol}\right)$, and $\mathrm{K}_{2} \mathrm{CO}_{3}(5.03 \mathrm{~g}, 36.3 \mathrm{mmol})$ in DMF $(15 \mathrm{~mL})$ was stirred at $80{ }^{\circ} \mathrm{C}$ for 24 hours. The resulting mixture was poured into water and extracted with ethyl acetate. The combined extracts were washed with a saturated $\mathrm{NH}_{4} \mathrm{Cl}$ aqueous solution and brine, dried over $\mathrm{MgSO}_{4}$, filtered, and the filtrate was evaporated. The residue was purified by column chromatography (eluent: $\mathrm{CHCl}_{3} /$ methanol $=10 / 1$ ) to obtain the desired compound $(3.77 \mathrm{~g}, 10.7 \mathrm{mmol})$ in $77 \%$ yield as a white solid. ${ }^{1} \mathrm{H}$ NMR $(400 \mathrm{MHz}): \delta=$ $1.43-1.66(\mathrm{~m}, 6 \mathrm{H}), 1.79-1.86(\mathrm{~m}, 2 \mathrm{H}), 3.65-3.70(\mathrm{~m}, 2 \mathrm{H}), 4.00(\mathrm{t}, J=6.6 \mathrm{~Hz}, 2 \mathrm{H}), 6.96(\mathrm{~d}, J$ $=8.8 \mathrm{~Hz}, 2 \mathrm{H}), 7.41(\mathrm{~d}, J=8.3 \mathrm{~Hz}, 2 \mathrm{H}), 7.47(\mathrm{~d}, J=8.8 \mathrm{~Hz}, 2 \mathrm{H}), 7.53(\mathrm{~d}, J=8.3 \mathrm{~Hz}, 2 \mathrm{H}) .{ }^{13} \mathrm{C}$ NMR $(100 \mathrm{MHz}): \delta=25.5,25.9,29.2,32.7,62.9,67.9,114.8,120.7,127.9,128.3,131.8$, 
132.3, 139.7, 158.9. Elemental analysis: Calcd.(\%) for $\mathrm{C}_{18} \mathrm{H}_{21} \mathrm{BrO}_{2}$ : C, 61.90; $\mathrm{H}, 6.06$. Found: C, 62.00; H, 6.12.

4-Bromo-4'-hexyloxybiphenyl. Quantities: 4-bromo-4'-hydroxybiphenyl (3.67 g, 14.7 mmol); 1-bromohexane (2.17 g, $13.1 \mathrm{mmol})$; TBAI (1.07 g, $2.90 \mathrm{mmol}) ; \mathrm{K}_{2} \mathrm{CO}_{3}$ (6.08 g, 44.0 mmol). The experimental procedure was as described for the preparation of 4-bromo-4'-(6-hydroxylhexyloxy)biphenyl. The crude product was purified by column chromatography (eluent: hexane/ethyl acetate $=20 / 1$ ) to give 4-bromo-4'-hexyloxybiphenyl $(3.72 \mathrm{~g}, 11.1 \mathrm{mmol})$ in $85 \%$ yield as a white solid. ${ }^{1} \mathrm{H}$ NMR $\left(\mathrm{CDCl}_{3}, 400 \mathrm{MHz}\right): \delta=0.91(\mathrm{t}, J$ $=7.1 \mathrm{~Hz}, 3 \mathrm{H}), 1.30-1.56(\mathrm{~m}, 6 \mathrm{H}), 1.76-1.83(\mathrm{~m}, 2 \mathrm{H}), 3.97(\mathrm{t}, J=6.6 \mathrm{~Hz}, 2 \mathrm{H}), 6.95(\mathrm{~d}, J=$ $8.8 \mathrm{~Hz}, 2 \mathrm{H}), 7.40(\mathrm{~d}, J=8.8 \mathrm{~Hz}, 2 \mathrm{H}), 7.46(\mathrm{~d}, J=8.8 \mathrm{~Hz}, 2 \mathrm{H}), 7.51(\mathrm{~d}, J=8.3 \mathrm{~Hz}, 2 \mathrm{H}) .{ }^{13} \mathrm{C}$ NMR $\left(\mathrm{CDCl}_{3}, 100 \mathrm{MHz}\right): \delta=14.1,22.6,25.7,29.2,31.6,68.1,114.8,120.7,127.9,128.2$, 131.7, 132.2, 139.7, 158.9. Elemental analysis: Calcd.(\%) for $\mathrm{C}_{18} \mathrm{H}_{21} \mathrm{BrO}$ : C, 64.87; H, 6.35. Found: C, 65.04; H, 6.26.

2,3-Difluoro-4-\{4-[4-(6-hydroxylhexyloxy)phenyl]phenyl\}phenol. A mixture of 4-bromo-4'-(6-hydroxylhexyloxy)biphenyl (1.04 g, $2.98 \quad \mathrm{mmol})$, 4-tert-butyldimethylsilyloxy-2,3-difluorophenylboronic acid $\left(9.53 \times 10^{-1} \mathrm{~g}, 3.77 \mathrm{mmol}\right)$, tetrakis(triphenylphosphine) palladium(0) $\left(\mathrm{Pd}\left(\mathrm{PPh}_{3}\right)_{4}\right)\left(6.50 \times 10^{-1} \mathrm{~g}, 5.60 \times 10^{-1} \mathrm{mmol}\right)$ in benzene $(80 \mathrm{~mL})$, methanol $(20 \mathrm{~mL})$, and a $2 \mathrm{M}$ sodium carbonate aqueous solution $(5 \mathrm{~mL})$ was heated to reflux and stirred for 2 hours. The reaction mixture was poured into a saturated $\mathrm{NH}_{4} \mathrm{Cl}$ aqueous solution and extracted with diethyl ether. The resulting extracts were washed with brine, dried over $\mathrm{MgSO}_{4}$, filtered, and the filtrate was evaporated. The residue was purified by column chromatography (eluent: hexane/ethyl acetate $=2 / 1$ ) to give 2,3-difluoro-4-\{4-[4-(6-hydroxylhexyloxy)phenyl]phenyl\}phenol $\left(8.08 \times 10^{-1} \mathrm{~g}, 2.03 \mathrm{mmol}\right)$ in $68 \%$ yield as a white solid. ${ }^{1} \mathrm{H}$ NMR (DMSO- $\left.d_{6}, 400 \mathrm{MHz}\right): \delta=1.36-1.49(\mathrm{~m}, 6 \mathrm{H}), 1.73(\mathrm{t}$, $J=6.8 \mathrm{~Hz}, 2 \mathrm{H}), 3.40(\mathrm{t}, J=5.9 \mathrm{~Hz}, 2 \mathrm{H}), 4.01(\mathrm{t}, J=6.4 \mathrm{~Hz}, 2 \mathrm{H}), 4.36(\mathrm{t}, J=5.1 \mathrm{~Hz}, 2 \mathrm{H})$, $6.87-6.92(\mathrm{~m}, 1 \mathrm{H}), 7.02(\mathrm{~d}, J=8.8 \mathrm{~Hz}, 2 \mathrm{H}), 7.17-7.21(\mathrm{~m}, 1 \mathrm{H}), 7.56(\mathrm{~d}, J=8.3 \mathrm{~Hz}, 2 \mathrm{H}), 7.64$ (d, $J=8.8 \mathrm{~Hz}, 2 \mathrm{H}), 7.70$ (d, $J=8.8 \mathrm{~Hz}, 2 \mathrm{H}), 10.53$ (s). ${ }^{13} \mathrm{C}$ NMR (DMSO- $\left.d_{6}, 100 \mathrm{MHz}\right): \delta=$ $25.2(\mathrm{~s}), 25.3$ (s), 28.6 (s), 32.4 (s), 60.6 (s), 67.5 (s), 113.1 (s), 114.9 (s), 119.7 (d, J=10 Hz), 123.8 (dd, $J=4.0,4.0 \mathrm{~Hz}$ ), 126.2 (s), 127.5 (s), 128.7 (d, $J=2.9 \mathrm{~Hz}), 131.6$ (s), 132.7 (s), 138.8 (s), 140.0 (dd, $J=145,242 \mathrm{~Hz}), 145.9$ (dd, $J=2.6,9.4 \mathrm{~Hz}), 148.2$ (dd, $J=11,246 \mathrm{~Hz}$ ), 158.4 (s). Elemental analysis: Calcd.(\%) for $\mathrm{C}_{24} \mathrm{H}_{24} \mathrm{~F}_{2} \mathrm{O}_{7}$ : C, 72.35; H, 6.07. Found: C, 72.20; H, 5.99 . 
4-tert-butyldimethylsilyloxy-2,3-difluorophenylboronic acid $(2.03 \mathrm{~g}, \quad 7.04 \mathrm{mmol})$; 4-bromo-4'-hexyloxybiphenyl (1.59 g, $4.77 \mathrm{mmol}) ; \operatorname{Pd}\left(\mathrm{PPh}_{3}\right)_{4}\left(1.94 \times 10^{-1} \mathrm{~g}, 1.60 \times 10^{-1}\right.$ mmol). The experimental procedure was as described for the preparation of 2,3-difluoro-4-\{4-[4-(6-hydroxylhexyloxy)phenyl]phenyl\}phenol. The crude product was purified by column chromatography (eluent: $\mathrm{CHCl}_{3} /$ ethyl acetate $=25 / 1$ ) to obtain 2,3-difluoro-4-\{4-[4-(hexyloxy)phenyl]phenyl\}phenol $\left(7.79 \times 10^{-1} \mathrm{~g}, 2.03 \mathrm{mmol}\right)$ in $42 \%$ yield as a white solid. ${ }^{1} \mathrm{H} \mathrm{NMR}\left(\mathrm{CDCl}_{3}, 400 \mathrm{MHz}\right): \delta=0.92(\mathrm{t}, J=6.8 \mathrm{~Hz}, 3 \mathrm{H}), 1.34-1.50(\mathrm{~m}$, $6 \mathrm{H}), 1.78-1.85(\mathrm{~m}, 2 \mathrm{H}), 4.01(\mathrm{t}, J=6.8 \mathrm{~Hz}, 2 \mathrm{H}), 5.23(\mathrm{~d}, J=3.9 \mathrm{~Hz}, 1 \mathrm{H}), 6.85-6.90(\mathrm{~m}, 1 \mathrm{H})$, $6.99(\mathrm{~d}, J=8.8 \mathrm{~Hz}, 2 \mathrm{H}), 7.12-7.16(\mathrm{~m}, 1 \mathrm{H}), 7.55(\mathrm{~d}, J=8.3 \mathrm{~Hz}, 2 \mathrm{H}), 7.56(\mathrm{~d}, J=8.8 \mathrm{~Hz}, 2 \mathrm{H})$, $7.63(\mathrm{~d}, J=8.3 \mathrm{~Hz}, 2 \mathrm{H}) .{ }^{13} \mathrm{C} \mathrm{NMR}\left(\mathrm{CDCl}_{3}, 100 \mathrm{MHz}\right): \delta=14.1$ (s), 22.6 (s), 25.7 (s), 29.2 (s), $31.5(\mathrm{~s}), 68.0(\mathrm{~s}), 113.0(\mathrm{~s}), 114.8(\mathrm{~s}), 120.8$ (d, $J=11 \mathrm{~Hz}), 123.6(\mathrm{dd}, J=3.9,3.9 \mathrm{~Hz}), 126.6$ (s), 127.3 (s), 129.0 (d, $J=2.7 \mathrm{~Hz}$ ), 132.7 (s), 133.5 (s), 139.7 (s), 140.8 (dd, $J=14,244 \mathrm{~Hz}$ ), 145.9 (dd, $J=2.5,9.9 \mathrm{~Hz}), 148.8$ (dd, $J=11,248 \mathrm{~Hz}), 158.8$ (s). Elemental analysis: Calcd.(\%) for $\mathrm{C}_{24} \mathrm{H}_{24} \mathrm{~F}_{2} \mathrm{O}_{2}$ : C, 75.37; H, 6.33. Found: C, 75.73; H, 6.12.

\section{6-(4-\{4-[2,3-Difluoro-4-(2-\{2-[2-(2-methoxyethoxy)ethoxy]ethoxy\}ethoxy)phenyl]pheny}

\section{l\}phenoxy)hexanol.}

A

solution

of

2,3-difluoro-4-\{4-[4-(6-hydroxylhexyloxy)phenyl]phenyl\}phenol $\left(9.10 \times 10^{-1} \mathrm{~g}, 2.28 \mathrm{mmol}\right)$, 2-\{2-[2-(2-methoxyethoxy)ethoxy] ethoxy\}ethyl $p$-tosylate $\left(7.50 \times 10^{-1} \mathrm{~g}, 2.07 \mathrm{mmol}\right)$, and $\mathrm{K}_{2} \mathrm{CO}_{3}\left(9.50 \times 10^{-1} \mathrm{~g}, 6.87 \mathrm{mmol}\right)$ in DMF $(10 \mathrm{~mL})$ was stirred at $80{ }^{\circ} \mathrm{C}$. After 16 hour of stirring, the cooled mixture was poured into water and extracted with ethyl acetate. The organic extracts were combined and washed with a saturated $\mathrm{NH}_{4} \mathrm{Cl}$ aqueous solution and brine, dried over $\mathrm{MgSO}_{4}$, filtered, and the filtrate was concentrated in vacuo. The residue was purified by column chromatography (eluent: ethyl acetate) to give 6-(4-\{4-[2,3-difluoro-4-(2-\{2-[2-(2-methoxyethoxy)ethoxy]ethoxy $\}$ ethoxy)phenyl]phenyl $\}$ ph enoxy)hexanol $\left(8.02 \times 10^{-1} \mathrm{~g}, 1.36 \mathrm{mmol}\right)$ in $66 \%$ yield as a white solid. ${ }^{1} \mathrm{H}$ NMR $\left(\mathrm{CDCl}_{3}\right.$, $400 \mathrm{MHz}): \delta=1.35-1.87(\mathrm{~m}, 9 \mathrm{H}), 3.38(\mathrm{~s}, 3 \mathrm{H}), 3.54-3.77(\mathrm{~m}, 14 \mathrm{H}), 3.91(\mathrm{t}, J=4.9 \mathrm{~Hz}, 2 \mathrm{H})$, $4.02(\mathrm{t}, J=6.6 \mathrm{~Hz}, 2 \mathrm{H}), 4.26(\mathrm{t}, J=4.6 \mathrm{~Hz}, 2 \mathrm{H}), 6.83-6.87(\mathrm{~m}, 1 \mathrm{H}), 6.98(\mathrm{~d}, J=8.8 \mathrm{~Hz}, 2 \mathrm{H})$, $7.12-7.17(\mathrm{~m}, 1 \mathrm{H}), 7.51-7.68(\mathrm{~m}, 6 \mathrm{H}) .{ }^{13} \mathrm{C} \mathrm{NMR}\left(\mathrm{CDCl}_{3}, 100 \mathrm{MHz}\right): \delta=25.5(\mathrm{~s}), 25.9(\mathrm{~s})$, $29.2(\mathrm{~s}), 32.6$ (s), 59.0 (s), 62.8 (s), 66.1 (s), 67.9 (s), 69.4 (s), 69.5 (s), 70.55 (s), 70.55 (s), 70.58 (s), 70.9 (s), 71.9 (s), 110.0 (s), 114.8 (s), 123.0 (d, $J=11 \mathrm{~Hz}), 123.4$ (dd, $J=4.6,4.6$ Hz), 126.7 (s), 128.0 (s), 129.0 (d, $J=3.7 \mathrm{~Hz}), 132.8$ (s), 133.0 (s), 140.1 (s), 141.8 (dd, $J=$ 
16, $248 \mathrm{~Hz}), 147.4$ (dd, $J=2.9,8.7 \mathrm{~Hz}), 148.8$ (dd, $J=11,249 \mathrm{~Hz}), 158.8$ (s). Elemental analysis: Calcd.(\%) for $\mathrm{C}_{33} \mathrm{H}_{42} \mathrm{~F}_{2} \mathrm{O}_{7}$ : C, 67.33; H, 7.19. Found: C, 67.43; H, 7.39.

\section{6-(4-\{4-[2,3-Difluoro-4-(2-\{2-[2-(2-methoxyethoxy)ethoxy]ethoxy\}ethoxy)phenyl]pheny} l\}phenoxy)hexanol monomethacrylate (M1). To a solution of 6-(4-\{4-[2,3-difluoro-4-(2-\{2-[2-(2-methoxyethoxy)ethoxy]ethoxy\}ethoxy)phenyl]phenyl $\}$-p henoxy)hexanol $\left(7.90 \times 10^{-1} \mathrm{~g}, 1.34 \mathrm{mmol}\right)$ and triethylamine $(1 \mathrm{~mL})$ in $\mathrm{CH}_{2} \mathrm{Cl}_{2}(15 \mathrm{~mL})$, 2,6-di-tert-butylphenol $\left(1.00 \times 10^{-3} \mathrm{~g}, 4.85 \times 10^{-3} \mathrm{mmol}\right)$ was added as a polymerization inhibitor. Methacryloyl chloride $\left(3.90 \times 10^{-1} \mathrm{~mL}, 4.03 \mathrm{mmol}\right)$ was added dropwise to the mixture with stirring at $0{ }^{\circ} \mathrm{C}$. The resultant mixture was stirred at room temperature for 3 hours in the dark to avoid unwanted polymerization. The reaction mixture was poured into water and extracted with $\mathrm{CHCl}_{3}$. The organic phase was separated and washed with a saturated $\mathrm{NH}_{4} \mathrm{Cl}$ aqueous solution and brine, dried over $\mathrm{MgSO}_{4}$, and filtered. The evaporation of the filtrate gave the crude product which was purified by column chromatography (eluent: $\left.\mathrm{CH}_{2} \mathrm{Cl}_{2}\right)$ to give $\mathbf{M 1}\left(7.04 \times 10^{-1} \mathrm{~g}, 1.07 \mathrm{mmol}\right)$ in $81 \%$ yield as a white solid. ${ }^{1} \mathrm{H}$ NMR $\left(\mathrm{CDCl}_{3}, 400 \mathrm{MHz}\right): \delta=1.47-1.58(\mathrm{~m}, 4 \mathrm{H}), 1.68-1.87(\mathrm{~m}, 4 \mathrm{H}), 1.95(\mathrm{~s}, 3 \mathrm{H}), 3.38(\mathrm{~s}, 3 \mathrm{H})$, 3.55-3.77 (m, 12H), $3.91(\mathrm{t}, J=4.6 \mathrm{~Hz}, 2 \mathrm{H}), 4.01(\mathrm{t}, J=6.3 \mathrm{~Hz}, 2 \mathrm{H}), 4.17(\mathrm{t}, J=6.4 \mathrm{~Hz}, 2 \mathrm{H})$, $4.26(\mathrm{t}, J=4.9 \mathrm{~Hz}, 2 \mathrm{H}), 5.56(\mathrm{~s}, 1 \mathrm{H}), 6.11(\mathrm{~s}, 1 \mathrm{H}), 6.83-6.87(\mathrm{~m}, 1 \mathrm{H}), 6.98(\mathrm{~d}, J=8.3 \mathrm{~Hz}$, 2H), 7.12-7.16 (m, 1H), 7.55-7.57 (m, 4H), $7.63(\mathrm{~d}, J=7.8 \mathrm{~Hz}, 2 \mathrm{H}) .{ }^{13} \mathrm{C}$ NMR $\left(\mathrm{CDCl}_{3}, 100\right.$ MHz): $\delta=18.3(\mathrm{~s}), 25.7(\mathrm{~s}), 25.8(\mathrm{~s}), 28.5$ (s), 29.1 (s), $59.0(\mathrm{~s}), 64.6$ (s), 67.8 (s), $69.4(\mathrm{~s})$, 69.5 (s), 70.5 (s), 70.6 (s), 70.9 (s), 71.9 (s), 109.9 (s), 114.7 (s), 123.0 (d, J=11 Hz), 123.4 (dd, $J=4.4,4.4 \mathrm{~Hz}), 125.2$ (s), 126.7 (s), 128.0 (s), 129.0 (d, $J=2.5 \mathrm{~Hz}), 132.8$ (s), 133.0 (s), 136.4 (s), 140.1 (s), 141.8 (dd, $J=15,248 \mathrm{~Hz}), 147.4$ (dd, $J=3.3,8.7 \mathrm{~Hz}), 148.8$ (dd, $J=11$, $249 \mathrm{~Hz}$ ), 158.7 (s), 167.5 (s). Elemental analysis: Calcd.(\%) for $\mathrm{C}_{37} \mathrm{H}_{46} \mathrm{~F}_{2} \mathrm{O}_{8}: \mathrm{C}, 67.67 ; \mathrm{H}, 7.06$. Found: C, 67.37; H, 6.90.

\section{2-\{2-[2-(2-\{2,3-Difluoro-4-[4-(4-hexyloxyphenyl)phenyl]phenoxy\} ethoxy)ethoxy]ethoxy}

\}ethanol. A stirred mixture of 2,3-difluoro-4-(4-(4-(hexyloxy)phenyl)phenyl)phenol (1.07 g, $2.79 \mathrm{mmol}), 2-\left\{2-[2-(2-h y d r o x y e t h o x y)\right.$ ethoxy] ethoxy $\}$ ethyl $p$-tosylate $\left(9.13 \times 10^{-1} \mathrm{~g}, 2.62\right.$ $\mathrm{mmol}), \mathrm{K}_{2} \mathrm{CO}_{3}(1.17 \mathrm{~g}, 8.46 \mathrm{mmol})$ in DMF $(15 \mathrm{~mL})$ was heated for 48 hours after which the cooled mixture was poured into water. The product was extracted into ethyl acetate, the organic extract was washed with a saturated $\mathrm{NH}_{4} \mathrm{Cl}$ aqueous solution and with brine, dried over $\mathrm{MgSO}_{4}$, and the solvent was evaporated. The crude product was purified by column chromatography (eluent: ethyl acetate $/ \mathrm{CHCl}_{3}=4 / 1$ ) to afford 2-\{2-[2-(2-\{2,3-difluoro-4-[4-(4-hexyloxyphenyl)phenyl]phenoxy $\}$ ethoxy)ethoxy]ethoxy $\}$ eth 
anol $(1.31 \mathrm{~g}, 2.34 \mathrm{mmol})$ in $89 \%$ yield as a white solid. ${ }^{1} \mathrm{H} \mathrm{NMR}\left(\mathrm{CDCl}_{3}, 400 \mathrm{MHz}\right): \delta=0.92$ (t, $J=6.8 \mathrm{~Hz}, 3 \mathrm{H}), 1.33-1.52(\mathrm{~m}, 6 \mathrm{H}), 1.77-1.84(\mathrm{~m}, 2 \mathrm{H}), 2.70(\mathrm{~s}, 1 \mathrm{H}), 3.61-3.78(\mathrm{~m}, 12 \mathrm{H})$, $3.91(\mathrm{t}, J=4.9 \mathrm{~Hz}, 2 \mathrm{H}), 4.00(\mathrm{t}, J=6.4 \mathrm{~Hz}, 2 \mathrm{H}), 4.26(\mathrm{t}, J=4.9,2 \mathrm{H}), 6.83-6.87(\mathrm{~m}, 1 \mathrm{H}), 6.98$ $(\mathrm{d}, J=8.8,2 \mathrm{H}), 7.12-7.16(\mathrm{~m}, 1 \mathrm{H}), 7.55-7.64(\mathrm{~m}, 6 \mathrm{H}) .{ }^{13} \mathrm{C} \mathrm{NMR}\left(\mathrm{CDCl}_{3}, 100 \mathrm{MHz}\right): \delta=$ 14.0 (s), 22.6 (s), 25.7 (s), 29.2 (s), 31.6 (s), 61.7 (s), 68.0 (s), 69.4 (s) 69.5 (s), 70.3 (s), 70.5 (s), 70.6 (s), 70.9 (s), 72.5 (s), 110.0 (s), 114.8 (s), 123.1 (d, $J=11 \mathrm{~Hz}), 123.5$ (dd, $J=4.0,4.0$ Hz), 126.7 (s), 128.0 (s), 129.0 (d, $J=2.9 \mathrm{~Hz}$ ), 132.7 (s), 133.0 (s), 140.1 (s), 141.8 (dd, $J=$ 15, $248 \mathrm{~Hz}), 147.4$ (dd, $J=2.6,8.3 \mathrm{~Hz}), 148.8$ (dd, $J=11,249 \mathrm{~Hz}), 158.8$ (s). Elemental analysis: Calcd.(\%) for $\mathrm{C}_{36} \mathrm{H}_{44} \mathrm{~F}_{2} \mathrm{O}_{7}$ : C, 68.80; H, 7.22. Found: C, 68.59; H, 7.19.

\section{2-\{2-[2-(2-\{2,3-Difluoro-4-[4-(4-hexyloxyphenyl)phenyl]phenoxy\} ethoxy)ethoxy]ethoxy} \}ethanol monomethacrylate (M2). To a solution of 2-\{2-[2-(2-\{2,3-difluoro-4-[4-(4-hexyloxyphenyl)phenyl]phenoxy\}ethoxy)ethoxy]ethoxy\} eth anol $\left(8.40 \times 10^{-1} \mathrm{~g}, 1.50 \times 10^{-1} \mathrm{mmol}\right)$, triethylamine $(1 \mathrm{~mL})$, and 2,6-di-tert-butylphenol (1.00 mg, $\left.4.85 \times 10^{-3} \mathrm{mmol}\right)$ in $\mathrm{CH}_{2} \mathrm{Cl}_{2}(15 \mathrm{~mL})$, methacryloyl chloride $\left(3.00 \times 10^{-1} \mathrm{~mL}, 3.10\right.$ mmol) was added dropwise at $0{ }^{\circ} \mathrm{C}$ over 5 minutes with stirring. The mixture was then stirred at room temperature for 3 hours in a light resistant container. The reaction mixture was poured into water and extracted with $\mathrm{CHCl}_{3}$. The organic phase was separated and washed with a saturated $\mathrm{NH}_{4} \mathrm{Cl}$ aqueous solution and brine, dried over $\mathrm{MgSO}_{4}$, and filtered. The evaporation of the filtrate gave the crude product which was purified by column chromatography (eluent: $\mathrm{CH}_{2} \mathrm{Cl}_{2} /$ ethyl acetate $\left.=25 / 1\right)$ to give $\mathbf{M 2}\left(4.81 \times 10^{-1} \mathrm{~g}, 1.07 \mathrm{mmol}\right)$ in $51 \%$ yield as a white solid. ${ }^{1} \mathrm{H}$ NMR $\left(\mathrm{CDCl}_{3}, 400 \mathrm{MHz}\right): \delta=0.91(\mathrm{t}, J=7.1 \mathrm{~Hz}, 3 \mathrm{H})$, $1.31-1.54(\mathrm{~m}, 6 \mathrm{H}), 1.75-1.84(\mathrm{~m}, 2 \mathrm{H}), 1.91-1.98(\mathrm{~m}, 3 \mathrm{H}), 3.68-3.76(\mathrm{~m}, 10 \mathrm{H}), 3.91$ (t, $J=$ $4.6 \mathrm{~Hz}, 2 \mathrm{H}), 4.00(\mathrm{t}, J=6.6 \mathrm{~Hz}, 2 \mathrm{H}), 4.25(\mathrm{t}, J=4.6 \mathrm{~Hz}, 2 \mathrm{H}), 4.31(\mathrm{t}, J=4.9 \mathrm{~Hz}, 2 \mathrm{H}), 5.57$ (t, $J=1.5 \mathrm{~Hz}, 1 \mathrm{H}), 6.13(\mathrm{~s}, 1 \mathrm{H}), 6.84-6.86(\mathrm{~m}, 1 \mathrm{H}), 6.98(\mathrm{~d}, J=8.3 \mathrm{~Hz}, 2 \mathrm{H}), 7.13-7.16(\mathrm{~m}, 1 \mathrm{H})$, 7.54-7.57 (m, 6H), $7.62(\mathrm{~d}, J=8.3,2 \mathrm{H}) .{ }^{13} \mathrm{C} \mathrm{NMR}\left(\mathrm{CDCl}_{3}, 100 \mathrm{MHz}\right): \delta=14.0(\mathrm{~s}), 18.3(\mathrm{~s})$, $22.6(\mathrm{~s}), 25.7$ (s), 29.2 (s), 31.6 (s), 63.8 (s), 68.0 (s), 69.1 (s), 69.4 (s), 69.5 (s), 70.6 (s), 70.6 (s), 70.6 (s), 70.9 (s), 110.0 (s), 114.8 (s), 123.1 (d, $J=11 \mathrm{~Hz}), 123.4$ (dd, $J=3.8,3.8 \mathrm{~Hz})$, 125.7 (s), 126.7 (s), 128.0 (s), 129.0 (d, $J=2.9$ Hz), 132.7 (s), 133.0 (s), 136.1 (s), 140.1 (s), 141.8 (dd, $J=15,248 \mathrm{~Hz}), 147.4$ (dd, $J=2.7,8.3 \mathrm{~Hz}$ ), 148.8 (dd, $J=11,249 \mathrm{~Hz}$ ), 158.8 (s), 167.3 (s). Elemental analysis: Calcd.(\%) for $\mathrm{C}_{36} \mathrm{H}_{44} \mathrm{~F}_{2} \mathrm{O}_{7}$ : C, 68.99; H, 7.08. Found: C, 68.93; $\mathrm{H}, 6.91$. 
Scheme S1. Synthesis of ethylene oxide derivatives.

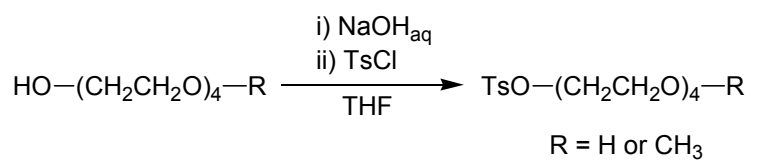

2-\{2-[2-(2-Methoxyethoxy)ethoxy]ethoxy\}ethyl p-tosylate. An aqueous solution of $\mathrm{NaOH}$ $(50 \%, 0.5 \mathrm{~mL})$ was slowly added to tetra(ethylene glycol) monomethyl ether $(2.37 \mathrm{~g}, 11.3$ mmol) with vigorously stirring at $0{ }^{\circ} \mathrm{C}$. After stirring for 30 minutes, a THF solution $(10 \mathrm{~mL})$ of $p$-toluenesulfonyl chloride $(\mathrm{TsCl})(2.60 \mathrm{~g}, 13.6 \mathrm{mmol})$ was slowly added to the reaction mixture. The solution was stirred at $0{ }^{\circ} \mathrm{C}$ for another 3 hours and then poured into water. The mixture was extracted with $\mathrm{CHCl}_{3}$, and the combined organic layers were washed with water and brine. After drying over $\mathrm{MgSO}_{4}$, the solvent was evaporated in vacuo. The residue was purified by column chromatography (eluent: ethyl acetate) to obtain 2-\{2-[2-(2-methoxyethoxy)ethoxy] ethoxy $\}$ ethyl $p$-tosylate $(2.61 \mathrm{~g}, 7.21 \mathrm{mmol})$ in $64 \%$ yield as a colorless oil. ${ }^{1} \mathrm{H}$ NMR $\left(\mathrm{CDCl}_{3}, 400 \mathrm{MHz}\right): \delta=2.45(\mathrm{~s}, 3 \mathrm{H}), 3.38(\mathrm{~s}, 3 \mathrm{H}), 3.54-3.70(\mathrm{~m}$, $14 \mathrm{H}), 4.16(\mathrm{t}, J=4.9 \mathrm{~Hz}, 2 \mathrm{H}), 7.35$ (d, $J=7.8 \mathrm{~Hz}, 2 \mathrm{H}), 7.80(\mathrm{~d}, J=7.8 \mathrm{~Hz}, 2 \mathrm{H}) .{ }^{13} \mathrm{C} \mathrm{NMR}$ $\left(\mathrm{CDCl}_{3}, 100 \mathrm{MHz}\right): \delta=21.5,58.9,68.5,69.2,70.4,70.4,70.6,71.8,127.8,129.7,132.8$, 144.7 .

Spectral data were consistent with those reported in the literature. ${ }^{\text {1a }}$

2- $\{2$-[2-(2-Hydroxyethoxy)ethoxy]ethoxy $\}$ ethyl $\boldsymbol{p}$-tosylate. To a cooled $\left(0{ }^{\circ} \mathrm{C}\right)$, vigorously stirred solution of tetraethylene glycol $(39.1 \mathrm{~g}, 112 \mathrm{mmol})$ in THF $(5 \mathrm{~mL})$, a $50 \% \mathrm{NaOH}$ aqueous solution ( $4 \mathrm{~mL}$ ) was slowly added. After 30 minutes of stirring, a THF solution of $\mathrm{TsCl}$ (3.90 g, $20.5 \mathrm{mmol})$ was slowly added to the mixture. The solution was stirred for 2 hours and then poured into water. The resultant mixture was extracted with $\mathrm{CHCl}_{3}$ and the organic phases were washed with a saturated $\mathrm{NaCl}$ aqueous solution. After drying over $\mathrm{MgSO}_{4}$, the solvent was removed by evaporation. The residue was purified by column chromatography (eluent: ethyl acetate) to afford 2-\{2-[2-(2-hydroxyethoxy)ethoxy] ethoxy ethyl $p$-tosylate $(5.42 \mathrm{~g}, 15.8 \mathrm{mmol})$ in $77 \%$ yield as a colorless oil. ${ }^{1} \mathrm{H} \mathrm{NMR}\left(\mathrm{CDCl}_{3}\right.$, $400 \mathrm{MHz}): \delta=2.47(\mathrm{~s}), 3.52-3.72(\mathrm{~m}, 14 \mathrm{H}), 4.15(\mathrm{t}, J=4.9 \mathrm{~Hz}, 2 \mathrm{H}), 7.33(\mathrm{~d}, J=8.3 \mathrm{~Hz}, 2 \mathrm{H})$, $7.88(\mathrm{~d}, J=8.3 \mathrm{~Hz}, 2 \mathrm{H}) .{ }^{13} \mathrm{C} \mathrm{NMR}\left(\mathrm{CDCl}_{3}, 100 \mathrm{MHz}\right): \delta=22.3,62.3,67.7,69.3,69.9,70.9$, $71.0,71.3,72.94,128.6,130.5,133.5,145.5$.

Spectral data were consistent with those reported in the literature. ${ }^{1 \mathrm{~b}}$ 
Sample preparation for in situ photopolymerization. After the addition of adequate amounts of lithium triflate $\left(\mathrm{LiOSO}_{2} \mathrm{CF}_{3}\right)$ in THF to each monomer, the solvent was evaporated slowly in vacuo to give lithium salt complexes. Then a solution of 2,2-dimethoxy-2-phenylacetophenone in dichloromethane ( $0.5 \mathrm{wt} \%$ to the $\mathrm{LC}$ monomers) was added to the complexes, the solvent was evaporated and dried in vacuo for 1 day at room temperature. All drying processes were carried out in the dark to avoid unwanted photopolymerization.

Photopolymerization. UV irradiation for the oriented LC complexes was performed by high-pressure mercury lamp (365 $\mathrm{nm}, 30 \mathrm{~mW} \mathrm{~cm}^{-2}$ for $\left.20 \mathrm{~min}\right)$. The polymerizable samples were placed in a pair of substrates (glass or ITO) and quenched from the isotropic phases to the smectic A phases. After the confirmation of homeotropic alignment by polarized micrographs, the cells were exposed to UV light at $5 \sim 6{ }^{\circ} \mathrm{C}$ above the smectic A-smectic $\mathrm{C}$ phase transition temperatures $\left(60{ }^{\circ} \mathrm{C}\right.$ for $\mathbf{M 1} / \mathrm{LiOSO}_{2} \mathrm{CF}_{3}$ and $80{ }^{\circ} \mathrm{C}$ for $\left.\mathbf{M 2} / \mathrm{LiOSO}_{2} \mathrm{CF}_{3}\right)$.

Preparation of P3. Non LC polymer $\mathbf{P 3}$ was obtained by free radical polymerization of the corresponding monomer in dry THF, using 2,2'-azobis(isobutyronitrile) as the initiator. The average molecular weight and polydispersity of the polymer were measured with a gel permeation chromatography calibrated with polystyrene standard in $\mathrm{CHCl}_{3}$. The weight-average molecular weight and polydispersity of $\mathbf{P 3}$ are $1.1 \times 10^{5}$ and 2.3 , respectively.

FE-SEM measurements. FE-SEM measurements were performed on a HITACHI S-900 at an accelerating voltage of $10 \mathrm{kV}$ for the oriented liquid crystalline polymers. The obtained samples of polymer films were peeled off from the substrates and the fractured surfaces of the films were shaded with platinum. 
(a)

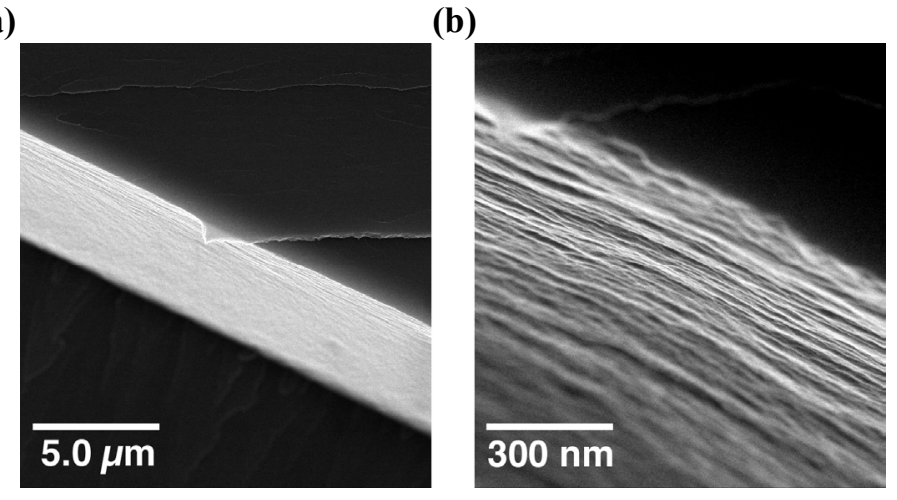

Figure S1. FE-SEM images of the macroscopically oriented complex $\mathbf{P 2} / \mathrm{LiOSO}_{2} \mathrm{CF}_{3}$. (a) Low magnified view of the edge of the film, (b) high magnified view of the image in (a).

Small angle $X$-ray scattering measurements and molecular conformation. The layer spacings of the films are 6.3 and $6.1 \mathrm{~nm}$ for $\mathbf{P 1} / \mathrm{LiOSO}_{2} \mathrm{CF}_{3}$ and $\mathbf{P 2} / \mathrm{LiOSO}_{2} \mathrm{CF}_{3}$, respectively (Figure S2), while the molecular conformations of monomers are 3.7 3.9 nm calculated under CVFF force field by means of the Discover3 module of MS modeling (version 3.1; Accelrys Inc.) (Figure S3). These results imply that the polymers form bilayer structures as illustrated in Figure 3.
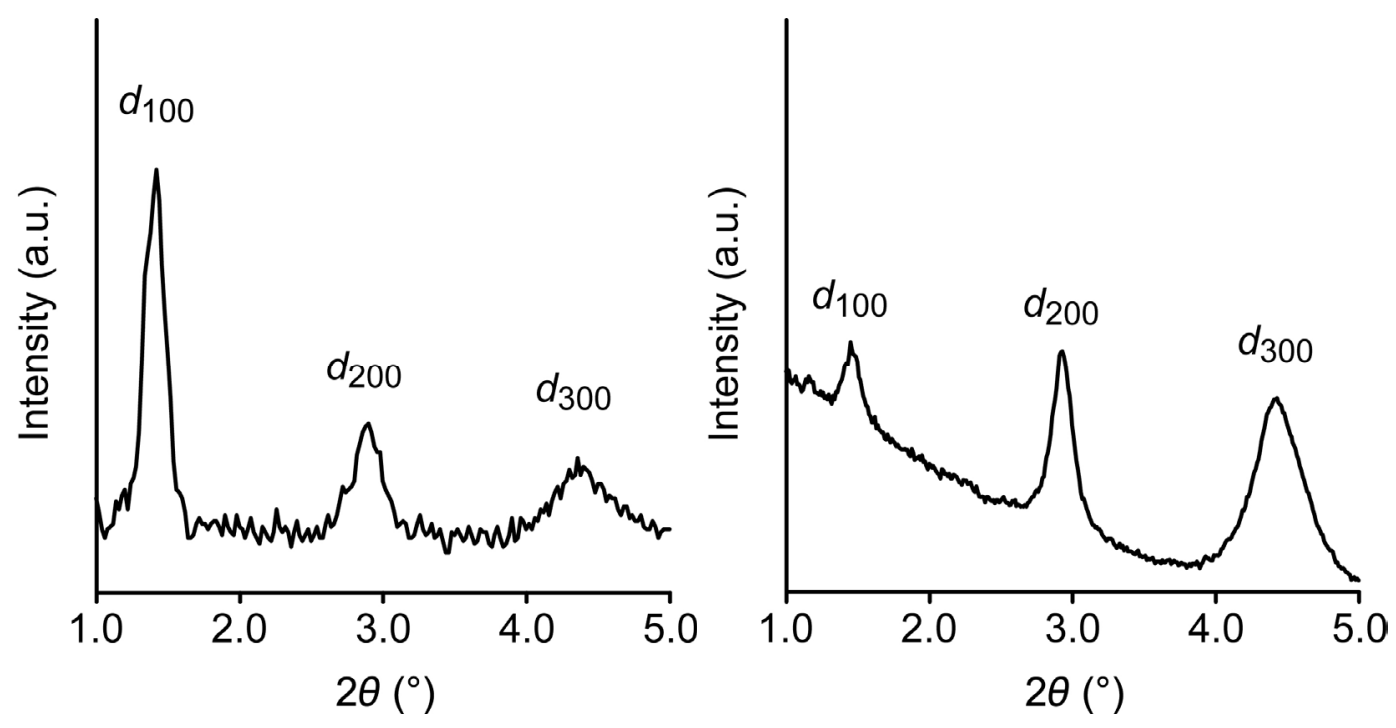

Figure S2. X-ray scattering patterns of $\mathbf{P 1} / \mathrm{LiOSO}_{2} \mathrm{CF}_{3}$ (left) and $\mathbf{P 2} / \mathrm{LiOSO}_{2} \mathrm{CF}_{3}$ (right). 


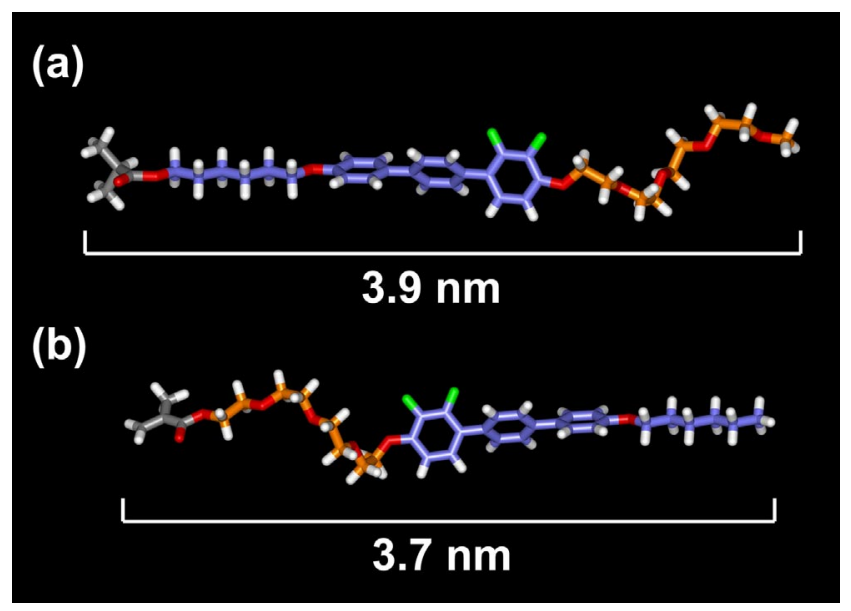

Figure S3. Molecular conformation of (a) M1 and (b) M2.

(a) Au electrode

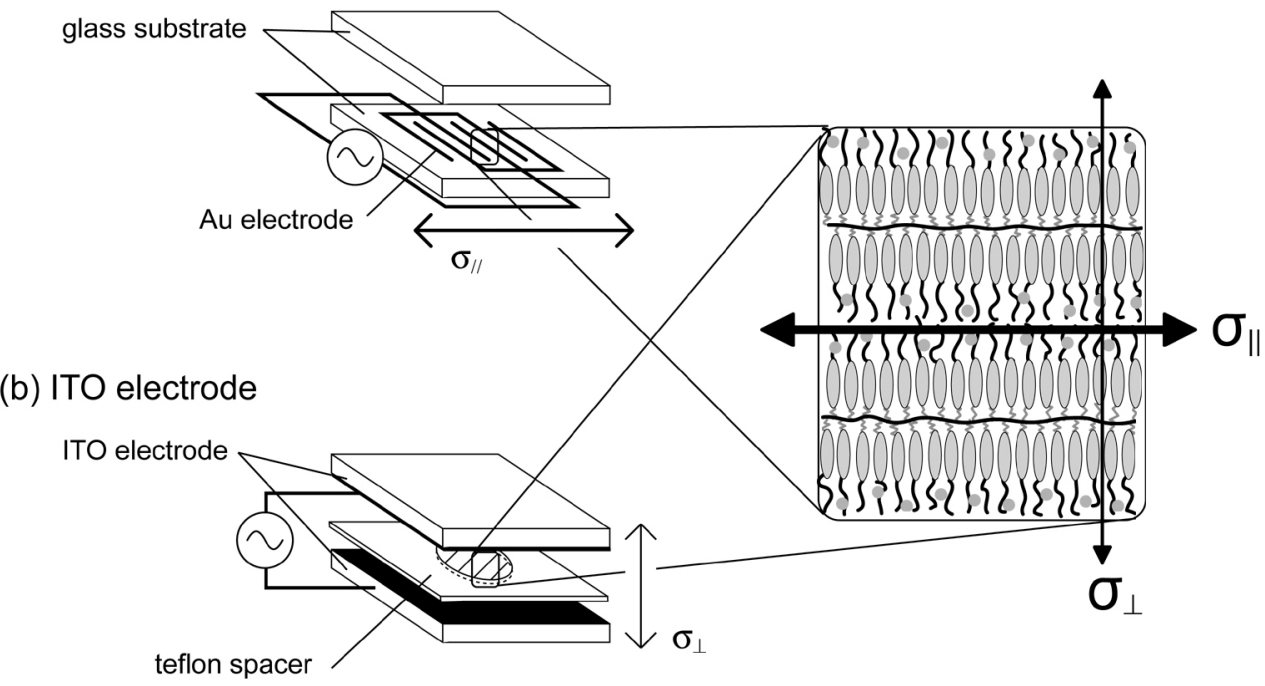

Figure S4. Schematic illustration of anisotropic ionic conductivity measurement cells for complex impedance methods. (a) Comb-shaped gold electrode cell for the measurements of the $\sigma_{\|}$values, (b) ITO substrate cell for the measurements of the $\sigma_{\perp}$ values.

\section{References.}

(1) (a) Brunsveld, L.; Zhang, H.; Glasbeek, M.; Vekemans, J. A. J. M.; Meijer, E. W. J. Am. Chem. Soc. 2000, 122, 6175-6182. (b) van Ameijde, J.; Liskamp, R. M. J. Org. Biomol. Chem. 2003, 1, 2661-2669. 